\title{
NEONATAL SEPSIS- ORGANISMS RESPONSIBLE AS DETECTED BY BLOOD CULTURE
}

\author{
Shyamali Datta1, Ravi Prakash Anand ${ }^{2}$, Panchanan Ghosh ${ }^{3}$
}

${ }^{1}$ Assistant Professor, Department of Paediatrics, Mata Gujri Memorial Medical College, Kishanganj, Bihar.

${ }^{2}$ Postgraduate Student, Department of Paediatrics, Mata Gujri Memorial Medical College, Kishanganj, Bihar.

3 Professor, Department of Paediatrics, Mata Gujri Memorial Medical College, Kishanganj, Bihar.

\section{ABSTRACT}

\section{BACKGROUND}

Neonatal sepsis refers to bacteraemia and clinical symptoms of infection caused by microorganisms and their toxic products in the first four weeks of life. It is the major cause of mortality and morbidity in neonates and accounts for $30 \%-50 \%$ of neonatal deaths in developing countries. In India incidence reported as 30 per 1000 live births for the year 2002 - 2003. Blood culture is the gold standard for diagnosis of neonatal sepsis apart from utility of routine haematological, biochemical and radiological tests. Now BacT/ALERT 3D microbial detection system are being used for blood culture.

This study will enable us to have important data regarding bacteriological spectrum associated with neonatal sepsis in suspected sepsis cases admitted in our rural hospital in Bihar.

\section{MATERIALS AND METHODS}

Our study included suspected cases of sepsis among all neonates delivered at this hospital and also included cases of neonatal sepsis referred from outside. We included 341 cases of neonates, which were suspected to have sepsis and in whom blood cultures were done.

\section{RESULTS}

Out of total 341 cases of suspected sepsis, 146 (42.8\%) showed positive isolates confirmed by blood culture. We had total 62 $(42.3 \%)$ Gram positive, 70 (48.1\%) Gram negative and $14(9.6 \%)$ fungal isolates out of total 146 positive blood culture isolates.

\section{CONCLUSION}

Our study showed that gram negative bacteria was the predominant cause of infection among neonates in our hospital. Coagulase negative Staphylococcus and Klebsiella were commonly isolated organisms among outborn and inborn neonates respectively.

\section{KEYWORDS}

Neonatal Sepsis, Organisms.

HOW TO CITE THIS ARTICLE: Datta S, Anand RP, Ghosh P. Neonatal sepsis- organisms responsible as detected by blood culture. J. Evolution Med. Dent. Sci. 2017;6(58):4327-4331, DOI: 10.14260/Jemds/2017/935

\section{BACKGROUND}

Neonatal sepsis refers to bacteraemia and clinical symptoms of infection caused by microorganism and their toxic products in the first four weeks of life.(1) It is the major cause of mortality and morbidity in neonates and accounts for $30 \%$ - $50 \%$ of neonatal deaths in developing countries.(2) In India, incidence reported as 30 per 1000 live births for the year 2002 - 2003.(3)

Since neonates is a relatively compromised host unable to localise infection, pathogenic bacteria gain access into blood causing generalised overwhelming infection (Septicaemia) or predominantly localised to lung (pneumonia) or involving meninges (Meningitis).(4) So systemic infections like septicaemia, pneumonia, meningitis, arthritis and osteomyelitis are considered as part of neonatal sepsis, but superficial infections like conjunctivitis or oral thrush are excluded from it.(5)

Financial or Other, Competing Interest: None.

Submission 01-06-2017, Peer Review 08-07-2017,

Acceptance 14-07-2017, Published 20-07-2017.

Corresponding Author:

Dr. Shyamali Datta,

\#60/129, H. P. Datta Lane,

Golf Gardens,

Kolkata-700033.

E-mail: data.shyamali@gmail.com

DOI: $10.14260 /$ jemds $/ 2017 / 935$
Based on time of onset of symptoms, neonatal sepsis is divided into two types, i.e. Early Onset Sepsis (EOS) if sepsis occurs in first 72 hours of life and Late Onset Sepsis (LOS) if it occurs after 72 hours of life.(6) Usually vertical transmission of pathogens causes EOS and horizontal transmission of pathogens causes LOS.(7) EOS is commonly present with respiratory distress and pneumonia, and source of infection is usually maternal genital tract. The risk factors of EOS include low birth weight $(<2500$ gms), prematurity, prolonged rupture of membrane, prolonged labour, frequent unclean vaginal examination, perinatal asphyxia (Apgar $<4$ at 1 minute). LOS is usually present with pneumonia or meningitis or both. The risk factors of LOS include low birth weight, prematurity, prolonged admission in NICU, poor hygiene, bottle feeding, etc.

The common symptoms and signs of neonatal sepsis are hypo/hyperthermia, refusal to feed, lethargy, poor cry, respiratory distress, brady/tachycardia, hypoglycaemia, hypotonia, metabolic acidosis, poor or absent neonatal reflex.(5) The bacterial agents implicated in early sepsis include E. coli; Group B streptococcus; coagulase negative staphylococcus; staphylo-aureus; Listeria monocytogenes. ${ }^{(7,8)}$ The organisms commonly associated with late onset sepsis include staphylo-aureus, coagulase negative staphylococcus, Klebsiella pneumonia, E. coli, Enterobacter spp., Pseudomonas aeruginosa and Acinetobacter spp.(8)

Blood culture is the gold standard for diagnosis of neonatal sepsis apart from utility of routine haematological, 
biochemical and radiological tests.(9) Now BacT/ALERT 3D microbial detection system are being used for blood cultures.(10) This advanced technique can detect bacteria even at a reduced concentration (1 - $2 \mathrm{CFU}$ per $\mathrm{mL}$ ) very early.(11) Along with sending blood culture, all neonates suspected of having sepsis must undergo sepsis screening which are total leukocyte count, absolute neutrophil count, immature to total neutrophil ratio, micro ESR and C-reactive protein. But these groups do not all require antimicrobial therapy.(12,13,14) Two or more abnormal sepsis screen parameters is considered positive sepsis screen and it warrants starting antibiotics. ${ }^{(15)}$ Negative sepsis screen along with persistence of clinical symptoms require repetition of sepsis screen after 12 hours. Further negative result almost exclude sepsis.

The bacteriological profile of causative agents of neonatal sepsis are different among developed and developing countries.(8) Even there is extensive regional variation in prevalence of bacterial agents within developing countries themselves.(8) Pattern of bacterial pathogens also changes with time.(7)

This study will enable us to have important data regarding bacteriological spectrum associated with neonatal sepsis in suspected sepsis cases admitted in a rural hospital in Bihar. It will help setting guidelines in choosing empirical therapy at this neonatal setup. This will be of immense help in reducing cost burden of treatment and lowering neonatal mortality from septicaemia in proposed geographic region.

\section{Aims and Objectives}

To find out the blood culture positivity of all suspected cases of neonatal sepsis among newborns delivered in this hospital and newborn referred from other health facilities and to isolate the organisms accountable for neonatal sepsis.

\section{MATERIALS AND METHODS}

\section{Study Type and Design}

This study was a cross-sectional study conducted in a rural medical college of Bihar in a span from March 2015 to March 2016.

\section{Study Area}

This study was carried out at level 2 and level 3 neonatal care units and post-partum ward of this hospital.

\section{Study Population}

Our study included suspected cases of sepsis among all neonates, which were delivered at this hospital. The study population also included suspected cases of neonatal sepsis referred to this hospital from outside.

\section{Sample Size}

We included 341 cases of neonates, which were suspected to have sepsis and in whom blood cultures were done. Parents and relatives were explained about nature of study and procedures. They were also assured about confidentiality of information and its anonymity.

\section{Exclusion Criteria}

Patient's refusal to participate in the study. Ethical Clearances
Written permission from Institutional Ethics Committee was obtained prior to beginning of the study. Parents or relative of the patients were explained in their own languages about nature of study and procedure. They were also assured about confidentiality of information and its anonymity.

\section{Study Tools}

1. Proforma for data collection.

2. Clinical examination.

3. Assessment of age by using New Ballard Score.

4. The blood culture was done by BacT/ALERT 3D and VITEK-2 Compact, which is an automated rapid culture system. It is proven to enhance recovery of organism from blood even if there has been prior antibiotic administration, which happened among neonates referred in this hospital from outside. Culture bottle contains peptone-enriched Tryptic Soy Broth (TSB) with Brain Heart Infusion (BHI) solid and activated charcoal. It detects $95 \%$ pathogens within 24 hours using specific colorimetric technology with visual positive signal.

\section{Study Technique}

Blood culture was taken from all neonates suspected to have sepsis. Sepsis was suspected in the following parameters-

1. All neonates of mother with fever during and before delivery. $\mathrm{H} / \mathrm{O}$ prolonged rupture of membrane for more than 24 hours, $\mathrm{H} / \mathrm{O}$ foul smelling or meconium stained liquor, $\mathrm{H} / \mathrm{O}$ more than 3 vaginal examinations.

2. Neonates having severe prematurity, H/O severe birth asphyxia with active resuscitation at time of birth.

3. Neonates with poor sucking reflex, respiratory distress, convulsion, hypo/hyperthermia, abdominal distension, bleeding manifestations with any of the above conditions, pathological jaundice diagnosed by clinical and laboratory features.

Parents and guardian of the patient were explained about the procedure and rationality of blood culture to be done in their own vernacular languages. They were explained about their right to opt out from the study at any time during the study after informing them about the blood culture to be an important component of treatment.

After location of vein selected accessible venepuncture site was scrubbed with $70 \%$ alcohol for minimum 30 seconds. Then Povidone iodine solution was applied in concentric circles centring the puncture site and then the site was allowed to dry.

Then the flip top overtap of the bottle was removed and it was cleansed with $70 \%$ alcohol. The bar code labelling of the bottle was kept intact; $3 \mathrm{~mL}$ of blood (in a ratio of 10 to 1 in culture bottle of $30 \mathrm{~mL}$ volume) was collected in a $5 \mathrm{~mL}$ syringe after inserting a intravenous cannula and the blood was directly inoculated into the bottle after inserting the needle into it. As contamination was highly possible during collection of blood for culture, utmost aseptic precautions were taken.

\section{Parameters Studied}

1. Positivity of culture.

2. Isolated organisms.

3. Organism pattern among inborn and outborn neonates. 


\section{Statistical Analysis}

We entered data in Microsoft Excel sheet 2010 and tabulated later. Frequency tables and cross tables have been constructed. Frequency along with percentage have been shown in tables. These are presented in Pie charts and Bar charts.

\section{RESULTS}

We had total 341 cases of neonates suspected of sepsis in our study, out of which 146 (42.8\%) showed positive isolates confirmed by blood culture and 195 (57.2\%) showed no growth. Out of 242 outborn 110 (45.6\%) showed positive growth, while 132 (54.4\%) had no growth in blood culture. Among 99 inborn 36 (36.2\%) showed positive growth, while $63(63.8 \%)$ had no growth in blood culture (Chart 1 ).

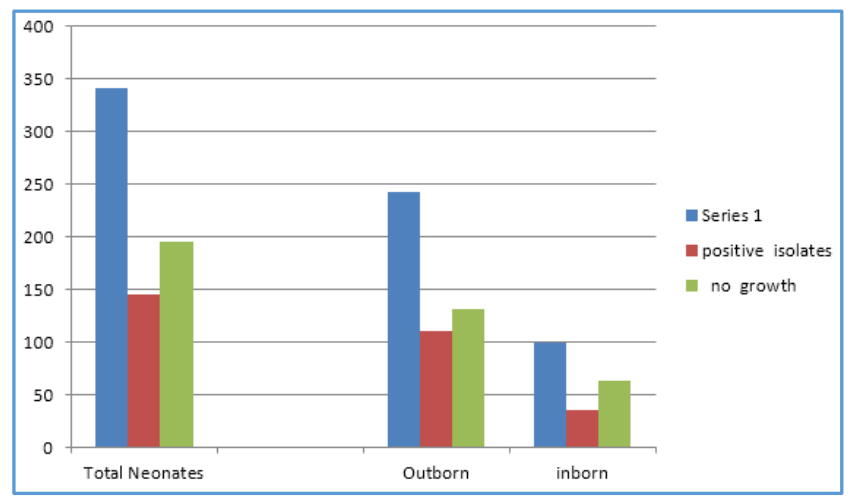

Chart 1. Showing Distribution of Blood Culture Results among Outborn/Inborn Neonates among total suspected Cases of Sepsis

\section{Distribution of Isolates}

We had total $62(42.3 \%)$ Gram positive, 70 (48.1\%) Gram negative and $14(9.6 \%)$ fungal isolates out of total 146 positive blood culture isolates. Among outborn showing positive blood cultures there was $53(48 \%)$ gram positive, 53 (48\%) gram negative and 5 (4\%) fungal isolates. Among inborn neonates showing positive growth in culture, there were $9(25 \%)$ cases Gram positive, 17 (48.6\%) gram negative and $9(26.4 \%)$ fungal isolates (Chart 2).

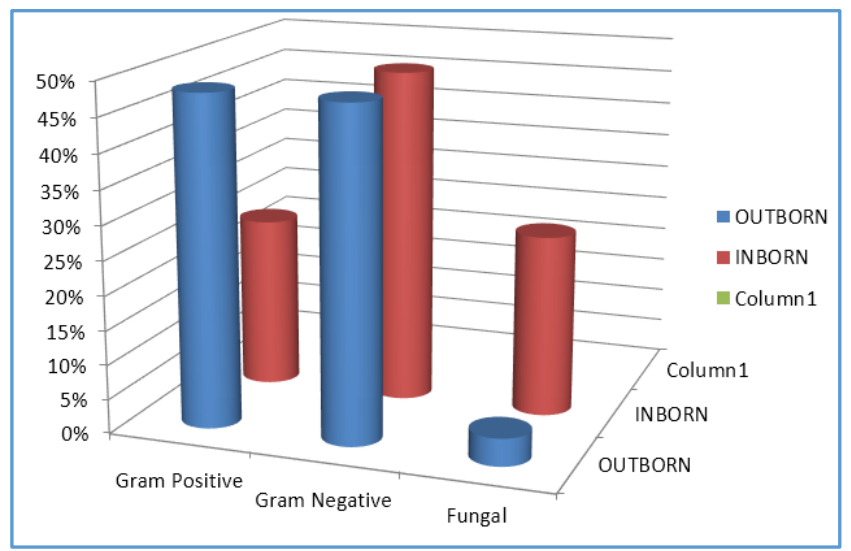

Chart 2. Distribution of various Organisms in Positive Blood Culture among Inborn and Outborn Neonates
We found 19.5\% (29 out of 146 isolates) early onset sepsis and $80.5 \%$ (117 out of 146 isolates) late onset sepsis among all culture confirmed cases. In outborns, early and late onset sepsis were $17.6 \%$ and $82.4 \%$ respectively confirmed by blood culture. Among inborn showing growth in culture, there were $25 \%$ and $75 \%$ respective cases of early and late onset sepsis.

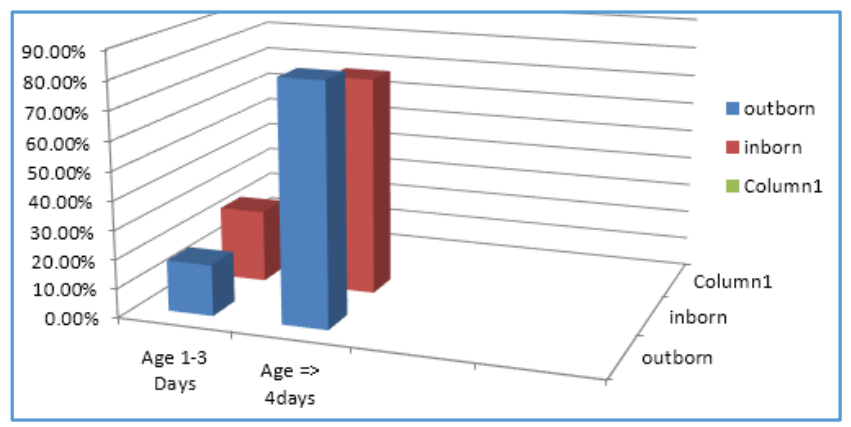

Chart 3. Distribution of Blood Culture Positive Early Onset Sepsis (Age 1 - 3 Days) and Late Onset

Positive Blood Culture Outborn, Inborn

Age Group in Days (1 - 3 days) 17.6\%, 25\%

Age Group in Days (> 4 days) $82.4 \%, 75 \%$

Sepsis (Age $=>4$ days) and Outborn $/$ Inborn neonates

\section{Pattern of Distribution of Different Organisms}

Coagulase negative staphylococcus (CONS) 34 cases (23.2\%) was the predominant isolates followed by Klebsiella 33 cases (22.2\%), E. coli 17 cases (11.6\%), Staph. aureus 15 (10.2\%), Candida 14 (9.6\%), Enterococcus 13 (8.9\%), Acinetobacter 8 (5.1\%), Burkholderia 7 (4.8\%) and Pseudomonas 7 (4.5\%) respectively. Out of all isolates among outborn babies, most predominant was CONS 28 cases (25.3\%) followed by Klebsiella 18 (16.7\%), E. coli 15 (14\%), Staph. aureus 13 (11.8\%), Enterococcus 12 (10.9\%), Acinetobacter 7 (6.3\%), Burkholderia 7 (6.3\%), Pseudomonas 5 (4.5\%) and Candida 5 (4\%). Among isolated organisms in Inborn, the most common was Klebsiella 15 (38.9\%) followed by Candida 9 (26.3\%), CONS 6 (16.7\%), Staph. aureus 2 (5.6\%), E. coli 2 (4.2\%), Pseudomonas 2 (4.2\%), Enterococcus 1 (2.8\%) and Acinetobacter 1 case.

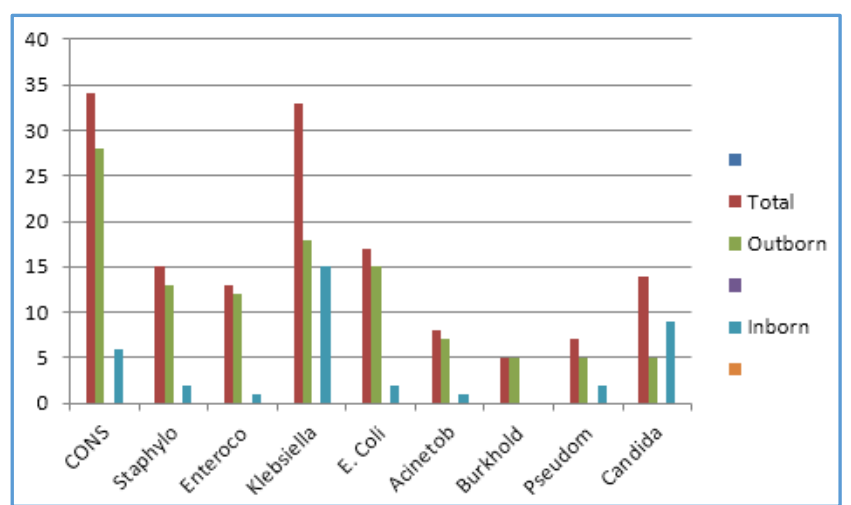

Chart 4. Showing Pattern of Distribution of Different Organisms 


\section{DISCUSSION}

Neonatal blood culture positivity rate varies among various studies done at different times and in different centres in the range $20 \%$ to $64 \% .^{(16,17,18,19,20)}$ Culture positivity around $20 \%$ was found in study by Baltimore et al, Haens et al,(21) Sharma et $\mathrm{al}^{(9)}$ using BacT/ALERT system. On the other hand culture positivity rate of as high as above $50 \%$ was found in study by Karthikeyan et al(22) and Tallur et $\mathrm{al}^{(23)}$ found culture positivity as high as $64 \%$ in his study in India.

In our study, we found blood culture positivity rate of $42.8 \%$ among all suspected neonates, which was similar to study by Thakur S et al(24) $42 \%$ in a study in North India and $41.6 \%$ culture positivity in a study in South India by Zakariya $\mathrm{BP}$ et al. But a wide variation of result was found in a study in central India, where Meher $\mathrm{V}$ et al(25) found only $22.1 \%$ culture positivity and Jyothi $\mathrm{P}$ et al(17) found only $19.2 \%$ culture positivity in their study in South India.

In our study out of total neonates with suspected sepsis $70.9 \%$ were outborn and $29.1 \%$ were inborn neonates which corroborates with study by Deepandra G et al(18) and by Samiya NK et al(7) in their studies as $77 \%$ and $75.4 \%$ outborn and $75.4 \%$ and $24.6 \%$ inborn neonates respectively.

The magnitude of neonatal sepsis in inborn neonates could be due to increased incidence of nosocomial sepsis from invasion of hospital flora colonising the skin into the bloodstream, uses of central and peripheral lines and indwelling catheters of the neonates during prolonged hospital stay, requirements of invasive ventilation, etc.

We found gram positive organism in $42.3 \%$ cases, gram negative in $48.1 \%$ cases and growth of Candida sp. in $9.6 \%$ cases out of total blood culture isolates. Predominance of growth of gram negative organisms also found by Lamba $\mathrm{M}$ et al(26) as $60.7 \%$ and Mehar $\mathrm{V}$ et al as $56.7 \%$ in their studies with suspected neonatal sepsis. Predominance of gram negative organism as high as $70 \%$ was found in a study by Rahaman et al.(27)

Fungal growth mainly candida was found in our study as $9.6 \%$ of total blood cultures. But increasing trend as high as $13.4 \%$ was found by study by Mehar $\mathrm{V}$ et al(27) and $22.3 \%$ among positive isolates in a study by Rao YK et al.(28) This increasing trend may be due to inappropriate use of antibiotics in hospital admitted neonates, prolonged hospital stay of low birth weight and very low birth weight neonates due to increased morbidity.

In our study, coagulase negative Staphylococcus was the most isolated pathogen $(23.2 \%)$ followed by Klebsiella pneumonia (22.2\%), Escherichia coli (11.6\% cases), Staphylococcus aureus (10.2\% cases), Candida species $(9.6 \%$ cases), Enterococcus faecium (8.9\% cases), Acinetobacter baumannii $(5.1 \%$ cases) and pseudomonas species $(4.4 \%$ cases). So most frequently encountered pathogens were coagulase negative Staphylococcus and Klebsiella pneumonia in our study, which is similar to a study in North India by Lamba $\mathrm{M}$ et al (52). But Movahedian $\mathrm{AH}$ et al(28) in their study found Pseudomonas aeruginosa (36\% cases) to be the most common isolated organism, which was in contrast to $4.4 \%$ of total isolates in our study. Shrestha $\mathrm{S}$ et al(16) and Jyothi $\mathrm{P}$ et al(18) in their studies found Klebsiella pneumonia was the predominant organism among the suspected cases. Escherichia coli $(66.7 \%)$ was the predominantly isolated organism in a study by Monsef $\mathrm{A}$ et al, which in contrast to our study where only $11.6 \%$ of total isolates were E. coli.
In our study, coagulase negative Staphylococcus and Klebsiella pneumonia were found in $25.3 \%$ and $16.7 \%$ cases among outborn neonates with suspected sepsis. These were two most frequently isolated organisms among outborn neonates, which is similar to studies by Samiya NK et al(7) and Deepandra et al.(18) But in contrast study by Amin AJ et al (8\%) found Klebsiella pneumonia (38.9\%) was the most frequently isolated organism among outborn neonates. Klebsiella pneumonia (38.9\%) and Candida (26.3\%) were the most frequently isolated organisms in inborn neonates in our study, which is similar to findings in the study by Anand JA et al(5) and Samiya NK et al.(7) Significant growth of Candida among inborn neonates in our study corroborates with study by Rao YK et al,(27) where they found Candida species as commonest organism in $22.3 \%$ of suspected sepsis cases.

The increased incidence of coagulase negative Staphylococcus among all the neonates suspected of having sepsis $23.2 \%$ of total isolates and $25.3 \%$ among culture positive outborn neonates) may be of equivocal significance. Because it is indicative of either contamination with skin flora or true bacteraemia. It is important that all the cases showing growth of coagulase negative Staphylococcus were also having increase in CRP level more than $10 \mathrm{mg} / \mathrm{dL}$ and most of them were outborn received in hospital after being treated outside with incomplete course of multiple antibiotics in most cases making neonates more susceptible to various blood stream infections with antibiotics resistant microorganisms. So the increased incidence of coagulase negative Staphylococcus in our study was probably true and it was not caused by contamination from skin flora.

\section{CONCLUSION}

Our study showed that gram negative bacteria was the predominant cause of infections among neonates in our hospital. Coagulase negative Staphylococcus and Klebsiella were commonly isolated organisms among outborn and inborn neonates respectively.

So continuous analysis of blood culture isolates and the pattern of their antimicrobial susceptibility by periodic epidemiological survey has become mandatory to recognise most frequently encountered organisms and to initiate safe and appropriate empirical antimicrobial therapy in a particular neonatal unit.

\section{REFERENCES}

[1] Srinivasa S, Arunkumar D. Bacterial isolates and their antibiotic susceptibility patterns in neonatal sepsis. Curr Pediatr Res 2014;18(2):83-6.

[2] Aggarwal R, Sarkar N, Deorari AK, et al. Sepsis in newborn. Indian J Pediatr 2001;68(12):1143-7.

[3] Report of national neonatal perinatal database. (National Neonatology Forum) 2002.

[4] Ghai OP, Gupta P, Paul VK. Ghai essential paediatrics. $6^{\text {th }}$ edn. New Delhi CBS Pub 2004:136-7.

[5] Amin AJ, Malam PP, Ansari PD, et al. Sensitivity and resistance pattern of antimicrobial agents used in cases of neonatal sepsis at a tertiary care centre in Western India. IJPSR 2016;7(7):3060-67.

[6] Singh M, Narang A, Bhakoo ON. Predictive perinatal score in the diagnosis of neonatal sepsis. J Trop Pediatr 1994;40(6):365-8. 
[7] Khan SN, Joseph S. Neonatal sepsis: antibiotic sensitivity \& resistance pattern of commonly isolated pathogens in a neonatal intensive care unit of a tertiary care hospital, south India. Int J Pharm Bio Sci 2012;3(4):802-9.

[8] Sawhney N, Shinu P, Singh VA. Bacteriological profile and antibiotic susceptibility pattern of neonatal septicaemia in a tertiary care hospital. Int J Curr Microbiol App Sci 2015;4(10):977-84.

[9] Sharma A, Kutty CVK, Sabharwal U, et al. Evaluation of sepsis screen for diagnosis of neonatal septicaemia. Indian J Pediatr 1993;60(4):559-63.

[10] Garcia-Prats JA, Cooper TR, Schneider VF, et al. Rapid detection of microorganism in the blood cultures of newborn infants by utilizing an automated blood culture system. Paediatrics 2000;105(3 Pt 1):523-7.

[11] Bhat RY, Baby LP. Early onset of neonatal sepsis: analysis of the risk factors and the bacterial isolates by using the Bac T alert system. JCDR 2011;5(7):1385-8.

[12] Escober GJ. The neonatal sepsis work up; personal reflections on the development of an evidence based approach towards newborn infections in a managed care organization. Paediatrics 1999;103(1 Suppl E):360-73.

[13] Polin RA, Geme JW. Neonatal sepsis. Advance Paediatr Infect Dis 1992;7:25-61.

[14] Riley LE, Celi AC, Onderdonk AB, et al. Association of epidural related fever and noninfectious inflammation in term labor. Obset Gynecol 2011;117(3):588-95.

[15] Garg D, Agarwal N. Aetiology and presentation of neonatal septicemia at teriary care hospital of South Rajasthan. IJMSE 2014;1(1):12-20.

[16] Shrestha S, Shrestha NC, Dongal SS, et al. Bacterial isolates and its antibiotic susceptibility pattern in NICU. Kathmandu Univ Med J 2013;11(41):66-70.

[17] Jyothi P, Basavaraj PV, Basavaraj MC. Bacteriological profile of neonatal septicemia and antibiotic susceptibility pattern of the isolates. J Nat Sci Biol Med 2013;4(2):306-9.
[18] English M, Ngama M, Mwalekwa L, et al. Signs of illness in Kenyan infants aged less than 60 days. Bull WHO 2004;82(5):323-9.

[19] Klingenberg C, Olomi R, Oneko $M$, et al. Neonatal morbidity and mortality in Tanzanian tertiary care hospital. Ann Trop Paediatr 2003;23(4):293-9.

[20] Mugalu J, Nakakeeto MK, Kiguli S, et al. Aetiology risk factors and immediate outcome of bacteriologically confirmed neonatal septicaemia in Mulago hospital, Uganda. Afr Health Sci 2006;6(2):120-6.

[21] Haens EJ, Roell-Schorer EAM, VAN-Elzakkar EPM, et al. Clinical availability of neonatal blood culture results at 48 hours. J Neonatal Perinatal Medicine 2009;2:10913.

[22] Karthikeyan G, Premkumar K. Neonatal sepsis: staphyalococcus aureus as the predominant pathogen. Indian J Pediatr 2001;68(8):715-7.

[23] Tallur SS, Kasturi AV, Nadgir SD, et al. clinicbacteriological study of neonatal septicaemia in Hubli. Indian J Paedia 2000;67(3):169-74.

[24] Thakur S, Sood A, Thakur K, et al. Bacteriological profile of neonatal septicaemia in a rural tertiary care hospital in North India. Indian J Med Microbiol 2016;34(1)67-71.

[25] Mehar V, Yadav D, Somani P, et al. Neonatal sepsis in a tertiary care center in central India: microbiological profile, antimicrobial sensitivity pattern and outcome. J Neonatal Perinatal Med 2013;6(2):165-72.

[26] Lamba M, Sharma D, Sharma R, et al Bacteriological spectrum and antimicrobial susceptibility pattern of neonatal septicaemia in a tertiary care hospital of North India. J Mater-Fetal Neonatal Med 2016;29(24):3993-8.

[27] Rao YK, Midha T, Garg A, et al. Neonatal septicaemia in North India due to extended spectrum beta lactamase producing gram negative bacteria. International $\mathrm{J}$ of Pharma \& Bio Science 2012;3(1):B282-90.

[28] Movahedian AH, Moniri R, Mosayebi Z. Bacterial culture of neonatal sepsis. Iranian J Publ Health 2006;35(4):84-9. 